\title{
Reliability Sensitivity Analysis in Stochastic Finite Element Models
}

\author{
Marcos A. Valdebenito \\ Associate Professor, Universidad Tecnica Federico Santa Maria, Dept. de Obras Civiles, \\ Valparaiso, Chile
}

Herman B. Hernández

Graduate Student, Universidad Tecnica Federico Santa Maria, Dept. de Obras Civiles, Valparaiso, Chile

Héctor A. Jensen

Professor, Universidad Tecnica Federico Santa Maria, Dept. de Obras Civiles, Valparaiso, Chile

\begin{abstract}
This paper proposes a framework for performing reliability sensitivity analysis of stochastic finite element models. The sensitivity measure corresponds to the derivative of the failure probability with respect to distribution parameters. The framework is cast as a post-processing step of Line Sampling and is formulated considering two different implementations. Numerical results suggest that both implementations are appropriate for estimating the sough probability sensitivity.
\end{abstract}

\section{INTRODUCTION}

Probability theory offers the possibility of accounting for the unavoidable effects of uncertainty on the performance of systems of engineering interest (Schuëller, 2006). Thus, the system's safety can be quantified in terms of, e.g. a failure probability. In such context, it is noted that the failure probability associated with a particular system depends on the selection of parameters that define a probability distribution (such as mean or variance). Hence, when analyzing a system, it is of interest evaluating not only the failure probability but also its sensitivity with respect to parameters of a probability distribution (Wu, 1994). The information on probability sensitivity is most useful for identifying the most influential parameters of a problem and can be used for, e.g. robust design (Schuëller and Jensen, 2008).

One possible means for evaluating the aforementioned sensitivity is calculating the partial derivative of the failure probability with respect to different parameters of the probability distributions that characterize the uncertainty of the structural model (Wu, 1994). While several approaches have been developed in the literature for performing reliability sensitivity analysis (see, e.g. Jensen et al. (2015); Marti (1995); Papaioannou et al. (2018); Wu (1994)), their application to problems involving spatial uncertainty remains mostly unexplored. Therefore, this contribution proposes an approach that allows estimating reliability sensitivity with respect to distribution parameters for stochastic finite element models. Such an approach is formulated within the framework of Line Sampling (Koutsourelakis et al., 2004), which is a simulationbased method for assessing failure probabilities. The proposed approach can be interpreted as a postprocessing step of a reliability analysis, i.e. the sought sensitivities are obtained as a byproduct of applying Line Sampling. In particular, this postprocessing step is implemented considering two different formulations based on numerical integration over the limit state function and the failure domain, respectively (Valdebenito et al., 2018). The 
performance of both approaches is discussed and compared by means of a numerical example.

\section{FORMULATION}

Consider a structural system that is represented by means of a linear static finite element model involving $N_{d}$ degrees-of-freedom (Bathe, 1996). In addition, a certain property of this structure is characterized by means of a weakly homogeneous isotropic log-normal random field. This property is represented through the discrete random variable vector $\boldsymbol{Y}$ of dimension $N_{y} \times 1$ whose probability density function is $f_{\boldsymbol{Y}}(\boldsymbol{y} \mid \boldsymbol{\theta})$, where $\boldsymbol{y}$ is a realization of the random variable vector and $\boldsymbol{\theta}$ is a vector collecting the parameters that describe the weakly homogeneous random field (i.e. mean, variance, correlation length). The equation that characterizes the performance of the system is:

$$
\boldsymbol{K}(\boldsymbol{y}) \boldsymbol{u}(\boldsymbol{y})=\boldsymbol{f}
$$

where $\boldsymbol{K}$ is a $N_{d} \times N_{d}$ stiffness matrix that depends on the realization of the random field $\boldsymbol{y} ; \boldsymbol{u}$ and $\boldsymbol{f}$ are vectors of dimension $N_{d} \times 1$ that represent displacements and external forces, respectively.

The weakly homogeneous random field is discretized considering the mid-point method and is represented applying the Karhunen-Loève expansion (see, e.g. Sudret (2000)). The discretized representation of the random field is equal to:

$$
\boldsymbol{y}=e^{\boldsymbol{a}+\boldsymbol{B} z}
$$

where vector $\boldsymbol{a}$ (of dimension $N_{y} \times 1$ ) and matrix $\boldsymbol{B}$ (of dimension $N_{y} \times N_{y}$ ) depend on the statistics of the log-normal random field and $z$ is a vector of independent, standard normal random variables of dimension $N_{y} \times 1$.

It is of interest controlling that a certain response of the structure $r(\boldsymbol{y})=\boldsymbol{\gamma}^{T} \boldsymbol{u}(\boldsymbol{y})$ does not exceed a prescribed threshold $b$, where $\boldsymbol{\gamma}$ is a $N_{D} \times 1$ vector of constant coefficients. Then, a performance function $g(\boldsymbol{y})=b-r(\boldsymbol{y})$ is defined and the probability of failure $p_{F}$ of the system is cast as follows (Ditlevsen and Madsen, 1996).

$$
p_{F}=\int_{g(\boldsymbol{y}) \leq 0} f_{\boldsymbol{Y}}(\boldsymbol{y} \mid \boldsymbol{\theta}) d \boldsymbol{y}
$$

A quick glance on eq. (3) reveals that the failure probability depends on vector $\boldsymbol{\theta}$ that contains the statistics of the random field. Clearly, it is of interest determining the sensitivity of the failure probability with respect to these statistics. This sensitivity can be measured in terms of, e.g. the partial derivative of the probability with respect to those statistics, as discussed in the following.

\section{PROBABILITY AND PROBABILITY SENSITIVITY ESTIMATION APPLY- ING MONTE CARLO SIMULATION}

Monte Carlo simulation has been widely applied for estimating both failure probability and its sensitivity with respect to distribution parameters (see, e.g. Papaioannou et al. (2013); Wu (1994)).

The application of Monte Carlo simulation (MCS) consists of generating $\mathrm{N}$ independent realizations of $\boldsymbol{y}$ distributed according to $f_{\boldsymbol{Y}}(\boldsymbol{y} \mid \boldsymbol{\theta})$. These samples are denoted as $\boldsymbol{y}^{(i)}, i=1, \ldots, N$. Then, the failure probability is estimated applying the following expression:

$$
p_{F} \approx \frac{1}{N} \sum_{i=1}^{N} I\left(g\left(y^{(i)}\right) \leq 0\right)
$$

where the indicator function $I(\cdot)$ is equal to 1 whenever the expression between parentheses holds and 0 , otherwise. Note that MCS can become numerically demanding when estimating small failure probabilities. In such cases, a large number of samples (proportional to $1 / p_{F}$ ) must be generated in order to produce an accurate estimator of the failure probability. Undoubtedly, this issue can pose a significant numerical burden. Nonetheless, MCS is applied in this work to benchmark the results produced by Line Sampling.

The probability sensitivities with respect to distribution parameters can be formulated by differentiating eq. (3).

$$
\frac{\partial p_{F}}{\partial \theta_{j}}=\int_{g(\boldsymbol{y}) \leq 0} \frac{\partial f_{\boldsymbol{Y}}(\boldsymbol{x} \mid \boldsymbol{\theta})}{\partial \theta_{j}} \frac{1}{f_{\boldsymbol{Y}}(\boldsymbol{y} \mid \boldsymbol{\theta})} f_{\boldsymbol{Y}}(\boldsymbol{y} \mid \boldsymbol{\theta}) d \boldsymbol{y}
$$

Following the MCS approach, this integral, representing the sensitivity of the failure probability, can 
be evaluated in terms the following estimator:

$$
\frac{\partial p_{F}}{\partial \theta_{j}} \approx \frac{1}{N} \sum_{i=1}^{N} I\left(g\left(\boldsymbol{y}^{(i)}\right) \leq 0\right) h_{\theta_{j}}\left(\boldsymbol{y}^{(i)}, \boldsymbol{\theta}\right)
$$

where, $h_{\theta_{j}}(\boldsymbol{y} \mid \boldsymbol{\theta})$ is a function defined as,

$$
h_{\theta_{j}}(\boldsymbol{y} \mid \boldsymbol{\theta})=\frac{\partial f_{\boldsymbol{Y}}(\boldsymbol{y} \mid \boldsymbol{\theta})}{\partial \theta_{j}} \frac{1}{f_{\boldsymbol{Y}}(\boldsymbol{y} \mid \boldsymbol{\theta})}
$$

For several cases of practical interest, the above equation can be reduced to a simple expression, as discussed in Wu (1994). In fact, for the case of a discrete log-normal random field, it can be shown that $h_{\theta_{j}}(\boldsymbol{y} \mid \boldsymbol{\theta})$ is equal to (see, e.g. Jensen et al. (2015)):

$$
\begin{aligned}
h_{\theta_{j}}(\boldsymbol{y} \mid \boldsymbol{\theta}) & =(\ln (\boldsymbol{y})-\boldsymbol{a})^{T}\left(\boldsymbol{\Sigma}^{-1}\right)^{T} \frac{\partial \boldsymbol{a}}{\partial \theta_{j}} \\
+ & \frac{1}{2}(\ln (\mathbf{y})-\boldsymbol{a})^{T}\left(\boldsymbol{\Sigma}^{-1}\right)^{T} \frac{\partial \boldsymbol{\Sigma}}{\partial \theta_{j}} \boldsymbol{\Sigma}^{-1} \\
& (\ln (\mathbf{y})-\boldsymbol{a})-\frac{1}{2} \operatorname{tr}\left(\boldsymbol{\Sigma}^{-1} \frac{\partial \boldsymbol{\Sigma}}{\partial \theta_{j}}\right)
\end{aligned}
$$

where $\Sigma$ is the covariance matrix of the underlying Gaussian field associated with the log-normal random field (see, e.g. Sudret (2000)). A salient aspect of the estimator in eq. (6) is that it can be applied using the same samples as those generated for assessing the failure probability. Hence, the estimation of the probability sensitivity can be seen as a post processing stage of the failure probability estimation that demands no additional structural analyses.

\section{PROBABILITY AND PROBABILITY SENSITIVITY ESTIMATION APPLY- ING LINE SAMPLING}

\subsection{Probability Estimation}

Line Sampling (see, e.g. Koutsourelakis et al. (2004)) is a simulation approach that allows estimating small failure probabilities efficiently with a great accuracy. In order to apply Line Sampling (LS), it is necessary to project the random variables of the reliability problem from the physical space to the standard normal space. This projection is denoted as $\boldsymbol{y}=T(z \mid \boldsymbol{\theta})$, where $T(\cdot)$ represents the transformation model and $z$ represents the vector of random variables in the standard normal space. For the specific case of this contribution, $T(\cdot)$ is given by eq. (2).

The first step involved in the application of LS is to identify the so-called important direction (which is denoted in the following as $\boldsymbol{\alpha}$ ). This direction is a unit vector that points towards the failure domain, i.e. the region in the standard normal space such that $g(T(\boldsymbol{z} \mid \boldsymbol{\theta})) \leq 0$. A schematic representation of $\boldsymbol{\alpha}$ in a two-dimensional space is illustrated in Figure 1. One possible way to find this important direction is setting it equal to the negative value of the gradient of the performance function at the origin of the standard normal space. For the sake of simplicity - and with no loss of generality - it is assumed in the following that the important direction is equal to $\boldsymbol{\alpha}=[1,0, \ldots, 0]^{T}$. Note that the selected direction according to this assumption can be achieved via a suitable coordinate transformation (in this case, rotation), since the standard normal probability density function $f_{\boldsymbol{Z}}(\boldsymbol{z})$ is rotationally invariant.

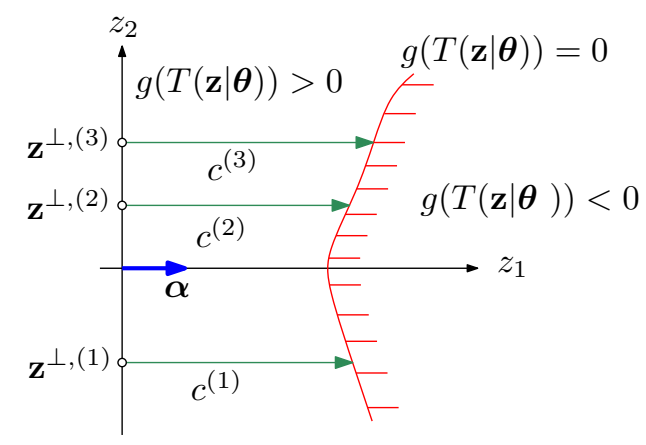

Figure 1: Schematic representation of Line Sampling.

Once the important direction has been defined, it is possible to identify a subset of the (standard normal) random variables of the problem contained in a $\left(N_{y}-1\right)$-dimensional hyperplane orthogonal to the important direction. This subset of random variables is termed as $z^{\perp}$. In view of the assumption that $\boldsymbol{\alpha}=[1,0, \ldots, 0]^{T}$, it is clear that $z^{\perp}=\left[z_{2}, \ldots, z_{N_{y}}\right]^{T}$. Taking into account the subset of random variables $z^{\perp}$, the probability integral in eq. (3) can be recast as:

$$
p_{F}=\int_{z^{\perp} \in \mathbb{R}^{N-1}} L\left(z^{\perp} \mid \boldsymbol{\theta}\right) f_{\boldsymbol{Z}^{\perp}}\left(z^{\perp}\right) d z^{\perp}
$$


where $f_{\boldsymbol{Z}^{\perp}}\left(\boldsymbol{z}^{\perp}\right)$ denotes the $\left(N_{y}-1\right)$-dimensional standard normal probability density function (pdf) and $L\left(\boldsymbol{z}^{\perp} \mid \boldsymbol{\theta}\right)$ is defined as:

$$
L\left(\boldsymbol{z}^{\perp} \mid \boldsymbol{\theta}\right)=\int_{g\left(T\left(\left(z_{1}, z^{\perp}\right)^{T} \mid \boldsymbol{\theta}\right)\right) \leq 0} f_{Z_{1}}\left(z_{1}\right) d z_{1}
$$

where $f_{Z_{1}}\left(z_{1}\right)$ denotes the one-dimensional standard normal pdf. Note that eq. (9) comprises a $\left(N_{y}-1\right)$-dimensional integral while eq. (10) comprises a one-dimensional integral. Hence, eq. (9) can be evaluated using direct MCS while eq. (10) can be solved by means of numerical integration (for a given realization of $z^{\perp}$ ). Such strategy is the basis of Line Sampling and is illustrated schematically in Figure 1. For a given sample $z^{\perp,(j)}$ (generated according to $f_{\mathbf{Z}^{\perp}}\left(\boldsymbol{z}^{\perp}\right)$ ), the one-dimensional line starting in $\boldsymbol{z}^{\perp,(j)}$ and along the direction $\boldsymbol{\alpha}$ is explored, in order to determine which portions of the line lie in the failure domain. In these portions, the one-dimensional normal standard pdf is integrated in order to estimate $L\left(z^{\perp,(j)} \mid \boldsymbol{\theta}\right)$, as indicated in eq. (10). Introducing the additional assumption that the aforementioned one-dimensional line crosses only once the limit state function $(g(T(z \mid \boldsymbol{\theta}))=0)$, eq. (10) simplifies to the following expression.

$$
\begin{aligned}
L\left(\boldsymbol{z}^{\perp,(i)} \mid \boldsymbol{\theta}\right) & =\int_{c^{(i)}}^{\infty} f_{Z_{1}}\left(z_{1}\right) d z_{1} \\
& =\Phi\left(-c^{(i)}\right)
\end{aligned}
$$

In the above equation, $\Phi(\cdot)$ represents the normal standard cumulative density function and $c^{(i)}$ is the distance between $z^{\perp,(i)}$ and the intercept between the associated line and the limit state function, as illustrated in Figure 1. The distance $c^{(i)}$ can be calculated according to the following procedure: (a) the performance function is evaluated over a coarse grid of $N_{L}$ points along the line being analyzed and then, (b) $c^{(i)}$ is determined by interpolation. Usually, this coarse grid comprises not more than 10 points $\left(N_{L} \leq 10\right)$. In summary, the application of Line Sampling involves the following steps: (1) First, the samples $z^{\perp,(i)}, i=1, \ldots, N$, distributed according to $f_{\boldsymbol{Z}^{\perp}}\left(\boldsymbol{z}^{\perp}\right)$, are generated. (2) Then, for each of these samples, the distance $c^{(i)}$ is calculated e.g. by interpolation. (3) Finally, the sought failure probability is estimated by means of the following expression.

$$
p_{F} \approx \frac{1}{N} \sum_{i=1}^{N} \Phi\left(-c^{(i)}\right)
$$

The total number of performance function evaluations (number of structural analyses) required for implementing Line Sampling is $N_{L} \times N$, i.e. for each of the $N$ lines being analyzed, a total of $N_{L}$ evaluations of the performance function must be carried out.

\subsection{Probability Sensitivity Estimation: Approach Based on Integration Over the Failure Do- main}

The sensitivities of the failure probability with respect to the input distribution parameters can be estimated based on eq. (5) which can be evaluated within the context of Line Sampling as follows. Assuming that an important direction $\boldsymbol{\alpha}=$ $[1,0, \ldots, 0]^{T}$ and the set of random variables $z^{\perp}$ have been identified, eq. (5) can be re-written as:

$$
\frac{\partial p_{F}}{\partial \theta_{j}}=\int_{\boldsymbol{z}^{\perp} \in \mathbb{R}^{N-1}} L_{j}^{*}\left(\boldsymbol{z}^{\perp} \mid \boldsymbol{\theta}\right) f_{\boldsymbol{Z}^{\perp}}\left(\boldsymbol{z}^{\perp}\right) d \boldsymbol{z}^{\perp}
$$

where $L_{j}^{*}\left(z^{\perp} \mid \boldsymbol{\theta}\right)$ is defined as:

$$
\begin{aligned}
& L_{j}^{*}\left(z^{\perp} \mid \boldsymbol{\theta}\right)= \\
& \int_{g\left(T\left(\left(z_{1}, z^{\perp}\right)^{T} \mid \boldsymbol{\theta}\right)\right) \leq 0} h_{\theta_{j}}^{*}\left(z_{1}, z^{\perp} \mid \boldsymbol{\theta}\right) f_{z_{1}}\left(z_{1}\right) d z_{1}
\end{aligned}
$$

and $h_{\theta_{j}}^{*}(\cdot)$ is defined as:

$$
h_{\theta_{j}}^{*}\left(z_{1}, z^{\perp} \mid \boldsymbol{\theta}\right)=h_{\theta_{j}}\left(T\left(\left(z_{1}, z^{\perp}\right)^{T} \mid \boldsymbol{\theta}\right), \boldsymbol{\theta}\right)
$$

Note that for the specific case of a log-normal random field, the above equation is equal to the analytic expression shown in eq. (8).

As for the case of the failure probability estimation with Line Sampling, eq. (13) can be evaluated by simply applying MCS. In addition, eq. (14) comprises an integral along a line that can be evaluated 
in closed form (see, e.g. Patel and Read (1996); Valdebenito et al. (2018)). Under the assumption that the line associated with the one-dimensional integral in eq. (14) crosses only once the limit state function $(g(T(z \mid \boldsymbol{\theta}))=0)$, the following estimator for the probability sensitivity is obtained.

$$
\frac{\partial p_{F}}{\partial \theta_{j}} \approx \frac{1}{N} \sum_{i=1}^{N} \int_{c^{(i)}}^{\infty} h_{\theta_{j}}^{*}\left(z_{1}, z^{\perp,(i)} \mid \boldsymbol{\theta}\right) f_{z_{1}}\left(z_{1}\right) d z_{1}
$$

An interesting feature of the estimator in eq. (16) is that it requires no additional performance function evaluations in case a reliability analysis with Line Sampling has been conducted previously. This is due to the fact that the distances $c^{(i)}, i=1, \ldots, N$ have already been found at the reliability analysis stage. Moreover, the one-dimensional integral contained in the estimator can be evaluated analytically (see, e.g. Patel and Read (1996); Valdebenito et al. (2018)), rendering the numerical cost associated with calculating eq. (16) negligible.

\subsection{Probability Sensitivity Estimation: Approach Based on Integration Over the Limit State Function}

An alternative approach for calculating the probability sensitivity within the context of Line Sampling can be formulated by directly evaluating the partial derivatives of eq. (9) with respect to the distribution parameter $\theta_{j}$.

$$
\frac{\partial p_{F}}{\partial \theta_{j}}=\int_{\boldsymbol{z}^{\perp} \in \mathbb{R}^{N_{y}-1}} \frac{\partial L\left(\boldsymbol{z}^{\perp} \mid \boldsymbol{\theta}\right)}{\partial \theta_{i}} f_{\boldsymbol{Z}^{\perp}}\left(\boldsymbol{z}^{\perp}\right) d \boldsymbol{z}^{\perp}
$$

where, $\partial L\left(z^{\perp} \mid \boldsymbol{\theta}\right) / \partial \theta_{j}$ is the partial derivative of the integral in eq. (10) with respect to $\theta_{j}$. As discussed previously, an estimator for this integral can be evaluated using MCS,

$$
\frac{\partial p_{F}}{\partial \theta_{j}} \approx \frac{1}{N} \sum_{i=1}^{N} \frac{\partial L\left(z^{\perp,(i)} \mid \boldsymbol{\theta}\right)}{\partial \theta_{j}}
$$

Again, assuming that the line associated with the one-dimensional integral $L\left(\boldsymbol{z}^{\perp,(i)} \mid \boldsymbol{\theta}\right)$ crosses only once the limit state function $(g(T(\boldsymbol{z} \mid \boldsymbol{\theta}))=0)$, the partial derivative $\partial L\left(\boldsymbol{z}^{\perp,(i)} \mid \boldsymbol{\theta}\right) / \partial \theta_{j}$ reduces to the following expression.

$$
\frac{\partial L\left(\boldsymbol{z}^{\perp,(i)} \mid \boldsymbol{\theta}\right)}{\partial \theta_{j}}=-f_{Z_{1}}\left(-c^{(i)}\right) \frac{\partial c^{(i)}}{\partial \theta_{j}}
$$

It can be shown (see, e.g. Papaioannou et al. (2013)) that the partial derivative of the distance $c^{(i)}$ with respect to $\theta_{j}$ can be expressed as,

$$
\frac{\partial c^{(i)}}{\partial \theta_{j}}=-\left.\frac{\left(\nabla_{z} g\right)^{T}\left(\partial z / \partial \theta_{j}\right)}{\left(\nabla_{z} g\right)^{T} \boldsymbol{\alpha}}\right|_{\boldsymbol{z}=\left\langle c^{(i)}, \boldsymbol{z}^{\perp,(i)}\right\rangle^{T}}
$$

In this equation, $\nabla_{z} g$ represents the gradient of the performance function (in the standard normal space). This gradient can be calculated analytically by differentiating eq. (1), see e.g. Haftka and Gürdal (1992). The partial derivative $\partial z / \partial \theta_{j}$ is calculated by differentiating eq. (2), yielding the following expression.

$$
\partial z / \partial \theta_{j}=-\boldsymbol{B}^{-1}\left(\frac{\partial \boldsymbol{a}}{\partial \theta_{j}}+\frac{\partial \boldsymbol{B}}{\partial \theta_{j}} z\right)
$$

From the above equation, it should be noted that $\partial \boldsymbol{a} / \partial \theta_{j}$ can be evaluated in closed form, see e.g. Jensen et al. (2015); on the contrary, the evaluation of $\partial \boldsymbol{B} / \partial \theta_{j}$ must be carried out numerically by performing an eigenvalue/eigenvector sensitivity analysis (Nelson, 1976), as the log-normal random field is represented applying the Karhunen-Loève expansion (see, e.g. Sudret (2000))

\section{EXAMPLE}

This example is adapted from Griffiths et al. (2013) and consists of a beam resting on clay that supports a point load, as depicted in Figure 2. The effect of the soil onto the beam is modeled considering a Winkler foundation. The beam has a length $L=12$ [m] and its bending stiffness is $E I=9500\left[\mathrm{kN} \mathrm{m}^{2}\right]$. The point load $P$ has a magnitude $P=28[\mathrm{kN}]$. The stiffness of the Winkler model is characterized as a weakly homogeneous log-normal random field with expected value $\mu=5800[\mathrm{kPa}]$ and standard deviation $\sigma=2900[\mathrm{kPa}]$ while the covariance is equal to $\sigma^{2} e^{-d^{2} / L_{C}^{2}}$, where $d$ denotes the Euclidean distance 


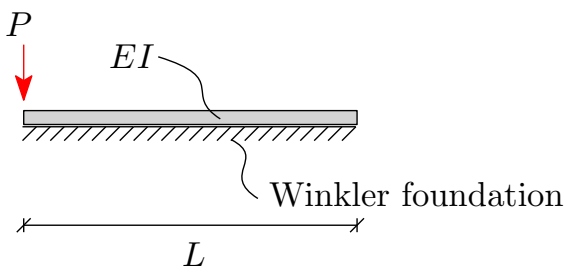

Figure 2: Beam resting on Winkler foundation

between two points and $L_{C}=6[\mathrm{~m}]$ is the correlation length. The response of interest is the vertical displacement of the beam beneath the point load and its threshold level is $b=20$ [mm]. The problem is modeled considering 10 beam elements of equal length. Such small model is selected in order to perform validation calculations applying Monte Carlo simulation.

The estimates of the probability sensitivity with respect to the expected value $\mu$ and variance $\sigma^{2}$ of the log-normal random field associated with the Winkler foundation model are shown in Figure 3. The probability sensitivity estimates generated with Line Sampling (considering a total of $N=10^{4}$ lines) applying both integration over the failure domain and integration over the limit state function are denoted as LS-IFD and LS-ILSF, respectively, while the results obtained applying Monte Carlo simulation (considering a total of $N=10^{7}$ samples) are denoted as MCS. The results obtained from the figure indicate that the two proposed implementations for probability sensitivity estimation applying Line Sampling converge to the same results produced by Monte Carlo simulation. Moreover, the proposed approach is highly efficient, as a low number of samples (lines) of Line Sampling is required in order to obtain accurate estimates of the sought sensitivity.

\section{CONCLUSIONS}

The results obtained in this contribution suggest that Line Sampling offers an appropriate means for estimating the sensitivity of the failure probability associated with models comprising random fields. Moreover, Line Sampling can be highly efficient from a numerical viewpoint.

While the results presented in this contribution are promising, further research is required on issues such as comparison of the two implementations
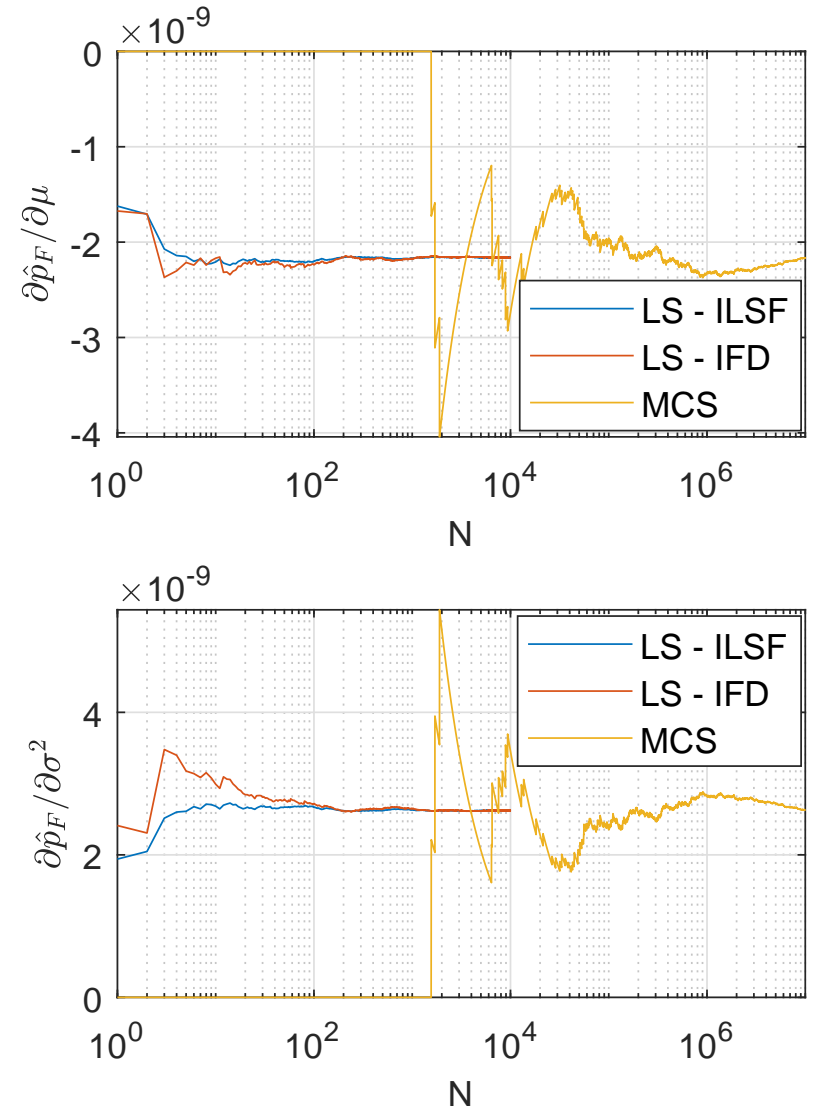

Figure 3: Estimates of Probability Sensitivity

based on Line Sampling for estimating probability sensitivity and testing on large scale stochastic finite element models. Such issues are already being investigated by the authors.

\section{ACKNOWLEDGMENTS}

This research is partially supported by CONICYT (National Commission for Scientific and Technological Research) under grant number 1180271. This support is gratefully acknowledged by the authors.

\section{REFERENCES}

Bathe, K. (1996). Finite Element Procedures. Prentice Hall, New Jersey.

Ditlevsen, O. and Madsen, H. (1996). Structural Reliability Methods. John Wiley and Sons.

Griffiths, D., Paiboon, J., Huang, J., and Fenton, G. (2013). "Reliability analysis of beams on random elastic foundations." Géotechnique, 63(2), 180-188. 
Haftka, R. and Gürdal, Z. (1992). Elements of Structural Optimization. Kluwer, Dordrecht, The Netherlands, $3^{\text {rd }}$ edition.

Jensen, H., Mayorga, F., and Valdebenito, M. (2015). "Reliability sensitivity estimation of nonlinear structural systems under stochastic excitation: A simulation-based approach." Computer Methods in Applied Mechanics and Engineering, 289, 1-23.

Koutsourelakis, P., Pradlwarter, H., and Schuëller, G. (2004). "Reliability of structures in high dimensions, part I: Algorithms and applications." Probabilistic Engineering Mechanics, 19(4), 409-417.

Liu, P. and Der Kiureghian, A. (1986). "Multivariate distribution models with prescribed marginals and covariances." Probabilistic Engineering Mechanics, 1(2), 105-112.

Marti, K. (1995). "Differentiation of probability functions: The transformation method." Computers \& Mathematics with Applications, 30(3-6), 361-382.

Nelson, R. (1976). "Simplified calculation of eigenvector derivatives." AIAA Journal, 14(9), 1201-1205.

Papaioannou, I., Breitung, K., and Straub, D. (2013). "Reliability sensitivity analysis with Monte Carlo methods." 11th International Conference on Structural Safety and Reliability (ICOSSAR), B. E. G. Deodatis and D. Frangopol, eds., New York, NY, USA, 5335-5342 (June 16-20).

Papaioannou, I., Breitung, K., and Straub, D. (2018). "Reliability sensitivity estimation with sequential importance sampling." Structural Safety, 75, 24 - 34.

Patel, J. and Read, C. (1996). Handbook of the normal distribution. Marcel Dekker Inc.

Schuëller, G. (2006). "Developments in stochastic structural mechanics." Archive of Applied Mechanics, 75(10-12), 755-773.

Schuëller, G. and Jensen, H. (2008). "Computational methods in optimization considering uncertainties An Overview." Computer Methods in Applied Mechanics and Engineering, 198(1), 2-13.

Sudret, B. (2000). "Stochastic finite element methods and reliability: A state-of-the-art report." Report no., University of California, Berkeley.
Valdebenito, M., Jensen, H., Hernández, H., and Mehrez, L. (2018). "Sensitivity estimation of failure probability applying line sampling." Reliability Engineering \& System Safety, 171, 99-111.

Wu, Y. (1994). "Computational methods for efficient structural reliability and reliability sensitivity analysis." AIAA Journal, 32(8), 1717-1723. 